\title{
Role of salvage radiotherapy for regional lymph node recurrence after radical surgery in advanced gastric cancer
}

\author{
Byoung Hyuck Kim, MD', Keun-Yong Eom, MD', Jae-Sung Kim, MD', \\ Hyung-Ho Kim, MD², Do Joong Park, MD² \\ Departments of ${ }^{1}$ Radiation Oncology and ${ }^{2}$ General Surgery, Seoul National University Bundang Hospital, Seongnam, Korea
}

Purpose: To evaluate the role of salvage radiotherapy (RT) for the treatment of regional lymph node recurrence (RLNR) after radical surgery in advanced gastric cancer.

Materials and Methods: We retrospectively analyzed medical records of 26 patients who underwent salvage treatment after diagnosis of RLNR between 2006 and 2011. Patients with peritoneal seeding or distant metastasis were excluded. Eighteen patients received RT with or without chemotherapy and the other 8 did chemotherapy only without RT. A three-dimensional conformal RT was performed with median dose of 56 Gy (range, 44 to $60 \mathrm{~Gy}$ ). Sixteen patients had fluoropyrimidine-based chemotherapy, 5 did taxane-based chemotherapy, and irinotecan was applied in 4.

Results: With a median follow-up of 20 months (range, 5 to 57 months), median overall survival (OS) and progression-free survival (PFS) after diagnosis of RLNR were 29 months and 12 months in the entire patients, respectively. Radiotherapy ( $p=0.007)$ and disease-free interval ( $p=0.033$ ) were statistically significant factors for OS in multivariate analysis. Median OS was 36 months in patients who received RT and 16 months in those who did not. Furthermore, delivery of RT ( $p<0.001)$, complete remission after salvage treatment $(p=0.040)$ and performance status $(p=0.023)$ were associated with a significantly better PFS. Gastrointestinal toxicities from RT were mild in most patients.

Conclusion: Salvage RT combined with systemic chemotherapy may be an effective treatment managing RLNR from advanced gastric cancer.

Keywords: Radiotherapy, Lymph nodes, Recurrence, Stomach neoplasms

\section{Introduction}

Gastric cancer occupies about 15\% of malignant tumors developed in Korea, the second most common malignancy following thyroid cancer [1]. Radical surgical resection of the primary site and regional lymph nodes (LNs) is considered as a standard treatment. Although increasing survival rate on the ground of advances in surgical techniques and adjuvant chemotherapy during recent decades, treatment outcomes remain unsatisfactory, as the five-year survival rate is approximately 65\% [2]. Gastric cancer is still the second leading cause of cancer mortality following lung cancer mainly due to high incidence and frequent relapse in spite of curative treatment [1].

LN metastasis at the time of surgery is one of the most significant prognostic factors for gastric cancer [3]. The multiple

Received 24 June 2013, Revised 25 July 2013, Accepted 13 August 2013.

Correspondence: Keun-Yong Eom, MD, Department of Radiation Oncology, Seoul National University Bundang Hospital, 82 Gumi-ro 173beon-gil, Bundang-gu, Seongnam 463-707, Korea. Tel: +82-31-787-7652, Fax: +82-31-787-4019, E-mail: 978sarang@daum.net

(c) This is an Open Access article distributed under the terms of the Creative Commons Attribution Non-Commercial License (http://creativecommons.org/ licenses/by-nc/3.0/) which permits unrestricted non-commercial use, distribution, and reproduction in any medium, provided the original work is properly cited.

www.e-roj.org 
Iymphatic drainage pathways around the stomach make it difficult to conduct complete LN dissection, which often results in regional $L N$ recurrence (RLNR) and contributes to further risks for distant metastasis. In the patterns of recurrence after curative resection, peritoneal seeding or hematogenous metastasis is regarded as major component, but locoregional recurrence also accounts for considerable part $[4,5]$.

Standard treatment of RLNR is not established [6]. As performing surgical dissection of RLNR is usually not preferred as a treatment option, systemic chemotherapy is often recommended in most of the patients. However, systemic chemotherapy alone is not regarded as effective for locoregional control $[5,7]$. In Korea, postoperative radiotherapy (RT) was not routinely used because of several limitations, although adjuvant chemoradiation after curative surgery significantly enhanced overall survival (OS) and relapse-free survival in prior Intergroup (INT)-0116 study [8]. Therefore salvage RT may play an important role as a safe and effective local treatment modality. We investigated the role of salvage RT for RLNR after radical surgery in advanced gastric cancer.

\section{Materials and Methods}

\section{Patient characteristics}

Current study analyzed gastric cancer patients with RLNR after radical surgery between January 2006 and December 2011 at the Seoul National University Bundang Hospital. In-hospital database search was undertaken to allow for retrieval of a large number of eligible patients. The search terms were "lymph node recurrence", "LN recurrence", "recurrent lesion", and "regional recurrence". Among the patients whose diagnosis was "stomach cancer" or "advanced gastric cancer", 43 patients satisfying following conditions were retrieved: any of the above search terms were written in their medical records or formal reading of follow-up imaging. Of these patients, 5 who did not received curative surgery, 4 with peritoneal seeding, and 8 with distant metastasis were excluded. As a result, total 26 patients were the subjects of this study. The medical records were reviewed retrospectively, and Institutional Review Board approval was obtained. All patients selected for our study underwent curative surgical resection, which was consisted of total or subtotal gastrectomy with R0 resection and D2 lymphadenectomy. Postoperative adjuvant chemotherapy was performed in 20 patients; 3 patients refused chemotherapy and the other 3 were not fit for chemotherapy because of poor performance status, stage IB, or belonging to observation group in clinical trial. Patients' performance status was assessed according to Eastern Cooperative Oncology Group (ECOG) performance score.

Of 26 patients, 18 patients (referred to as the RT group) were treated with RT with or without sequential chemotherapy and 8 (referred to as the no-RT group) received systemic chemotherapy only after diagnosis of RLNR. Whether to perform RT or not was at the discretion of treating physician based on the disease extent, performance status of the patient, and agreement of the patient and guardians. RLNRs were clinically diagnosed by imaging studies such as computed tomography (CT), abdominal ultrasonography (USG), or positron emission tomography (PET) without tissue diagnosis. If suspicious lesions were detected by USG or PET, contrastenhanced CT was routinely performed. RLNR is defined as a presence of regional LN with short diameter larger than $1.0 \mathrm{~cm}$ on $\mathrm{CT}$.

Sites of RLNR were classified into three levels according to the LN staging system by Japanese Gastric Cancer Association [9]. Perigastric LNs along the lesser and greater curvature of the stomach were defined as nodal region 1 (NR1). The nodes along the left gastric, common hepatic, celiac, splenic artery were defined as NR2. And more distant LNs along the hepatoduodenal ligament and the root of the mesentery, and in the para-aortic lesion were regarded as NR3. In case of multiple LN recurrences, maximal distance between LNs was calculated using $\mathrm{CT}$.

\section{Treatments}

1) Chemotherapy: Treatment sequences after the diagnosis of RLNR are shown in Table 1. There is heterogeneity of sequences of salvage treatment because the optimal policy is not defined. Most patients were recommended chemotherapy

Table 1. Treatment sequences

\begin{tabular}{lc}
\hline \multicolumn{1}{c}{ Sequence } & No. of patients \\
\hline $\mathrm{CT}$ & 8 \\
$\mathrm{CT} \rightarrow \mathrm{RT}$ & 5 \\
$\mathrm{CT} \rightarrow \mathrm{RT} \rightarrow \mathrm{CT}$ & 1 \\
$\mathrm{CT} \rightarrow \mathrm{CCRT}$ & 2 \\
$\mathrm{CT} \rightarrow \mathrm{CCRT} \rightarrow \mathrm{CT}$ & 4 \\
$\mathrm{CCRT}$ & 2 \\
$\mathrm{CCRT} \rightarrow \mathrm{CT}$ & 3 \\
$\mathrm{RT}$ & 1 \\
\hline
\end{tabular}

$\mathrm{CT}$, chemotherapy; RT, radiotherapy; CCRT, concurrent chemoradiotherapy. 
as a part of first salvage treatment option with or without $\mathrm{RT}$. Just one patient received RT alone due to patient's refusal of chemotherapy. Heterogeneous chemotherapy regimens were used depend on the medical oncologist's preference and patient's performance status. Sixteen patients had fluoropyrimidine-based chemotherapy (e.g., capecitabine plus oxaliplatin) and 5 did taxane-based chemotherapy (e.g. docetaxel plus cisplatin). Irinotecan was applied in 4 patients with concurrent chemoradiation. The timing of RT was also varied according to the patient's status and physician's judgment.

2) Radiotherapy: A three-dimensional conformal RT technique was used in all patients received RT. RT was conducted using a linear accelerator (Clinac 21EX; Varian Medical Systems, Palo Alto, CA, USA) with 6 or 15 MV photon beam. Median total dose was $56 \mathrm{~Gy}$ (range, 44 to $60 \mathrm{~Gy}$ ), with fractions of 1.8 to 2.0 Gy once daily, five times per week. The median dose to adjacent regional nodal areas was $45 \mathrm{~Gy}$ (range, 39.6 to $46 \mathrm{~Gy}$ ) and a boost dose of 9 to 20 Gy was delivered to gross tumor volume (GTV).

CT scan was performed for RT planning on supine position with both arms raised above the head. A scan thickness of $4 \mathrm{~mm}$ was used. GTV was defined as enlarged recurrent LNs. Clinical target volume was delineated around the GTV including adjacent nodal area at risk considering microscopic tumor extension. Planning target volume was defined as CTV plus 0.5 to $1.0 \mathrm{~cm}$ margin.

\section{Evaluation of response, survival, and toxicity}

Acute toxicities caused by chemotherapy were assessed by Common Terminology Criteria for Adverse Events (CTCAE) ver. 4.0. During radiation therapy, clinical evaluation of patients for toxicity was performed once weekly by radiation oncologists. Gastrointestinal toxicity was graded according to the Radiation Therapy Oncology Group (RTOG) acute radiation morbidity scoring criteria. The follow-up abdomen CT was performed in 1 month after completion of radiation therapy and repeated every 2 to 6 months thereafter. The patients who have not received $\mathrm{RT}$ were also followed up regularly with imaging studies every 2 to 6 months. Response evaluation of LNs was based on Response Evaluation Criteria in Solid Tumors (RECIST) ver. 1.1.

The sites of failure were classified as follows: the failure was regarded as local if progression was detected in the radiation field or enlargement of initial recurred LNs was identified for patients in no-RT group, and as abdominal if progression was detected in the peritoneal cavity (including the liver, intraabdominal LNs, and peritoneum). Progression-free survival (PFS) was defined as the time interval between the date of diagnosis of RLNR and any failure after salvage treatment. OS was calculated from the date of diagnosis of RLNR to the date of death from any cause or the last follow-up.

\section{Statistical analysis}

Pearson chi-square test or Fisher exact test was used to compare the distribution of clinical parameters between two groups. The OS and PFS were determined through the KaplanMeier method and compared using the log-rank test. For multivariate analysis, Cox proportional-hazards model were used to evaluate the correlation of OS or PFS with variables. Variables with p-value less than 0.1 in univariate analysis were entered into the backward stepwise regression model to adjust potential confounding effects. SPSS ver. 18.0.1 (SPSS Inc., Chicago, IL, USA) was used for statistical analyses.

\section{Results}

\section{Patients' characteristics}

Patient characteristics at the time of RLNR are shown in Table 2. There were 18 men (69.2\%) and 8 women (30.8\%) with a median age of 63 years (range, 34 to 80 years). ECOG performance status was 0 in 4 (15.4\%) patients, 1 in 17 (65.4\%) and 2 in 5 (19.2\%), respectively. Median disease-free interval between initial gastrectomy and RLNR was 12 months (range, 3 to 105 months). Six patients (23.1\%) had clinical symptoms such as abdominal pain or obstructive jaundice associated with RLNR. Isolated LN recurrence was observed in 13 patients (50.0\%), and the other had multiple (median 3, range 2 to 8 ) LNs recurrence. No LN recurrence was occurred in the site of NR1. Eight patients (30.8\%) were recurred in NR2 and 18 (69.2\%) in NR3. In the subgroup of patients with multiple LN recurrences, maximal distance between LNs was median $60 \mathrm{~mm}$ (range, 20 to $160 \mathrm{~mm}$ ). Diameter of recurrent LN was median $22 \mathrm{~mm}$ (range, 12 to $37 \mathrm{~mm}$ ).

\section{Response to salvage treatment}

After salvage treatment, contrast-enhanced CT scans were performed every 2 to 6 months. Maximal tumor regression during follow-up period was regarded as treatment response. Of 8 patients treated with chemotherapy alone, 1 achieved a complete remission (CR), 2 did a partial remission (PR), 3 
Table 2. Patient and tumor characteristics

\begin{tabular}{lc}
\hline \multicolumn{1}{c}{ Variable } & No. of patients \\
\hline Age (yr), median (range) & $63(34-80)$ \\
$<60$ & 8 \\
$\geq 60$ & 18 \\
Gender & \\
Male & 18 \\
Female & 8 \\
ECOG & \\
$0-1$ & 21 \\
2 & 5 \\
Disease-free interval (yr) & \\
$<1$ & 12 \\
$\geq 1$ & 14 \\
Symptoms & \\
Absent & 20 \\
Present & 6 \\
No. of recurrent LN & \\
1 & 13 \\
$\geq 2$ & 13 \\
Site of RLNR & \\
NR2 & \\
NR3 & \\
Diameter of recurrent LN (cm) & \\
$<2$ & 18 \\
$\geq 2$ & \\
Adjuvant chemotherapy after surgery & \\
Yes & \\
No & \\
Radiotherapy & \\
Yes & \\
No & \\
\hline & \\
\hline & \\
&
\end{tabular}

ECOG, Eastern Cooperative Oncology Group; LN, Iymph node; $R L N R$, regional lymph node recurrence; $N R$, nodal region.

did stable disease (SD), and 2 did progressive disease (PD). Twelve patients received chemotherapy first followed by RT or concurrent chemoradiotherapy. In these patients, $1 C R, 5$ $P R$, 4 SD, and 2 PD were achieved after chemotherapy (before RT) and then 4 CR, 4 PR, and 4 SD after RT. In 6 patients who underwent RT first with or without chemotherapy, 2 had a $C R$, 3 had a $P R$, and 1 had $P D$, respectively.

\section{Patterns of failure}

Of 7 patients with CR to salvage treatment, 3 were disease-free at the time of last follow-up. Four patients experienced disease recurrence in the para-aortic $L N$ and lung $(n=1)$, liver $(n=1)$, and mediastinal $L N(n=2)$, respectively. Among 19 patients with residual disease, tumor progression was observed in 17 including 12 with local failure. Local failure rate was $27.8 \%$ (5/18) in RT group and 87.5\% (7/8) in no-RT group. The site of progression was local and abdominal $(n=11)$, abdominal $(n=$ $1)$, local and the lung $(n=1)$, abdominal and the lung $(n=1)$, abdominal and the supraclavicular $\operatorname{LN}(n=1)$, the lung $(n=1)$, and the supraclavicular $L N(n=1)$, respectively.

\section{Survival and prognostic factors}

With a median follow-up of 20 months (range, 5 to 57 months), median OS and PFS were 29 months and 12 months, respectively. The results of univariate and multivariate analyses of OS and PFS are listed in Tables 3 and 4. Three factors appeared to be associated with OS in univariate analysis: disease-free interval, symptoms at the time of RLNR, and CR status after salvage treatment (Table 3). RT did not significantly affect $O S(p=0.076)$. After adjusting for disease-free interval, symptom, and CR status, there was significant difference in OS between patients who received RT and those treated with chemotherapy alone (hazards ratio $=0.193 ; 95 \%$ confidence interval, 0.059-0.637; $p=0.007$ ) (Table 4). Median OS was 36 months in patients received RT and 16 months in those who did not (Fig. 1). Disease-free interval was also associated with OS in multivariate analysis $(p=0.033$ ) (Table 4).

Furthermore, delivery of RT was associated with a significantly better PFS $(p<0.001)$ as was CR status $(p=0.040)$, and performance status $(p=0.023)$ (Table 3$)$. These variables remained as significant factors in multivariate analysis (Table 4). Median PFS was 20 months for RT group and 5 months for non-RT group (Fig. 1). All 5 patients without any failure were treated with RT meanwhile all patients treated without RT eventually progressed.

\section{Patients receiving upfront chemotherapy first without radiotherapy}

Twenty patients underwent chemotherapy as first line treatment in their salvage course, as shown in Table 1. Among them, 12 patients (60\%) were referred to radiation oncologist and received RT after several cycles of chemotherapy, whereas other 8 did not receive RT. All characteristics except ECOG status were not significantly different between patients received $R T$ and those who did not. The number of patients with ECOG 0-1 vs. 2 was 7 vs. 5 in RT group and 8 vs. 0 in nonRT group ( $p=0.055)$. The number of recurrent LNs, maximal distance between recurred LNs (in case of multiple LNs) or response to upfront chemotherapy were not significantly different between two groups (data not shown). The median 
Table 3. Univariate analysis for prognostic factors of survival

\begin{tabular}{|c|c|c|c|c|c|}
\hline Variable & No. of patients & $1-y r$ OS $(\%)$ & $p$-value & $1-y r$ PFS (\%) & $p$-value \\
\hline \multicolumn{6}{|l|}{ Age (yr) } \\
\hline$<60$ & 8 & 100.0 & \multirow[t]{2}{*}{0.331} & 62.5 & \multirow[t]{2}{*}{0.989} \\
\hline$\geq 60$ & 18 & 88.9 & & 60.6 & \\
\hline \multicolumn{6}{|l|}{ Gender } \\
\hline Male & 18 & 88.9 & \multirow[t]{2}{*}{0.419} & 55.6 & \multirow[t]{2}{*}{0.139} \\
\hline Female & 8 & 100.0 & & 75.0 & \\
\hline \multicolumn{6}{|l|}{ ECOG } \\
\hline $0-1$ & 21 & 90.5 & \multirow[t]{2}{*}{0.348} & 51.9 & \multirow[t]{2}{*}{0.023} \\
\hline 2 & 5 & 100.0 & & 100.0 & \\
\hline \multicolumn{6}{|l|}{ Disease-free interval (yr) } \\
\hline$<1$ & 12 & 83.3 & \multirow[t]{2}{*}{0.015} & 66.7 & \multirow[t]{2}{*}{0.510} \\
\hline$\geq 1$ & 14 & 100.0 & & 57.1 & \\
\hline \multicolumn{6}{|l|}{ Symptoms } \\
\hline Absent & 20 & 95.0 & \multirow[t]{2}{*}{0.034} & 59.6 & \multirow[t]{2}{*}{0.431} \\
\hline Present & 6 & 83.3 & & 66.7 & \\
\hline \multicolumn{6}{|l|}{ No. of recurrent $L N$} \\
\hline 1 & 13 & 92.3 & \multirow[t]{2}{*}{0.613} & 69.2 & \multirow[t]{2}{*}{0.350} \\
\hline$\geq 2$ & 13 & 92.3 & & 52.7 & \\
\hline \multicolumn{6}{|l|}{ Site of RLNR } \\
\hline NR2 & 8 & 100.0 & \multirow[t]{2}{*}{0.702} & 75.0 & \multirow[t]{2}{*}{0.251} \\
\hline NR3 & 18 & 88.9 & & 55.6 & \\
\hline \multicolumn{6}{|c|}{ Diameter of recurrent LN (cm) } \\
\hline$<2$ & 12 & 83.3 & \multirow[t]{2}{*}{0.772} & 66.7 & \multirow[t]{2}{*}{0.807} \\
\hline$\geq 2$ & 14 & 100.0 & & 57.1 & \\
\hline \multicolumn{6}{|c|}{ Adjuvant chemotherapy after surgery } \\
\hline Yes & 20 & 90.0 & \multirow[t]{2}{*}{0.620} & 60.0 & \multirow[t]{2}{*}{0.950} \\
\hline No & 6 & 100.0 & & 66.7 & \\
\hline \multicolumn{6}{|l|}{ Radiotherapy } \\
\hline Yes & 18 & 94.4 & \multirow[t]{2}{*}{0.076} & 83.3 & 0.000 \\
\hline No & 8 & 87.5 & & 12.5 & \\
\hline CR status after salvage $t r$ & & & & & \\
\hline Yes & 7 & 100.0 & 0.040 & 83.3 & 0.040 \\
\hline No & 19 & 89.5 & & 46.8 & \\
\hline
\end{tabular}

OS, overall survival; PFS, progression-free survival; ECOG, Eastern Cooperative Oncology Group; LN, lymph node; RLNR, regional lymph node recurrence; $\mathrm{NR}$, nodal region; $\mathrm{CR}$, complete response.

Table 4. Multivariate analysis for overall survival and progression-free survival

\begin{tabular}{|c|c|c|c|c|c|c|}
\hline \multirow{2}{*}{ Variable } & \multicolumn{3}{|c|}{ Overall survival } & \multicolumn{3}{|c|}{ Progression-free survival } \\
\hline & $H R$ & $95 \% \mathrm{Cl}$ & $p$-value & $H R$ & $95 \% \mathrm{Cl}$ & $p$-value \\
\hline Disease-free interval ( $\geq 1$ yr vs. $<1$ yr) & 0.233 & $0.061-0.891$ & 0.033 & - & - & - \\
\hline Symptoms (absent vs. present) & 0.336 & $0.093-1.205$ & 0.094 & - & - & - \\
\hline Radiotherapy (yes vs. no) & 0.193 & $0.059-0.637$ & 0.007 & 0.112 & $0.026-0.481$ & 0.003 \\
\hline CR status (yes vs. no) & 0.449 & $0.089-2.257$ & 0.331 & 0.156 & $0.039-0.623$ & 0.009 \\
\hline Performance status (ECOG 0-1 vs. 2) & - & - & - & 8.353 & $1.579-44.195$ & 0.013 \\
\hline
\end{tabular}

$\mathrm{HR}$, hazard radio; $\mathrm{Cl}$, confidence interval; $\mathrm{CR}$, complete response; ECOG, Eastern Cooperative Oncology Group. 
A

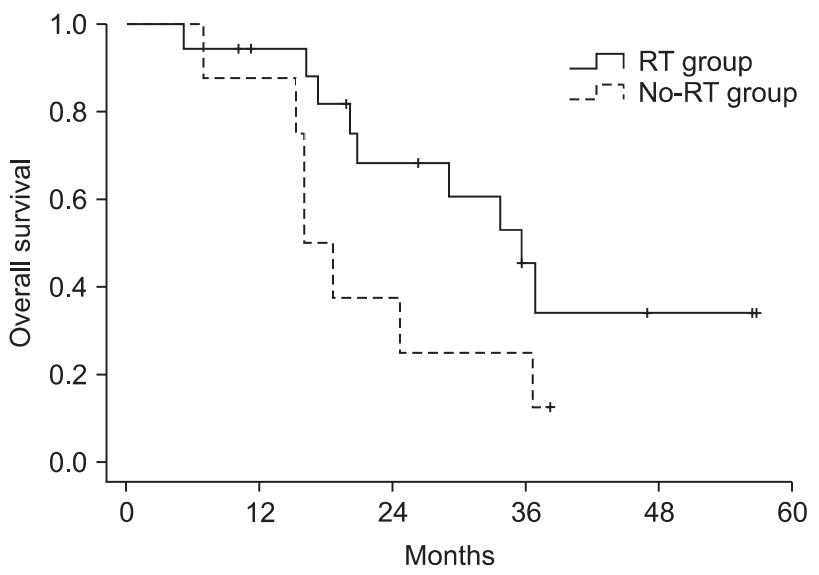

B

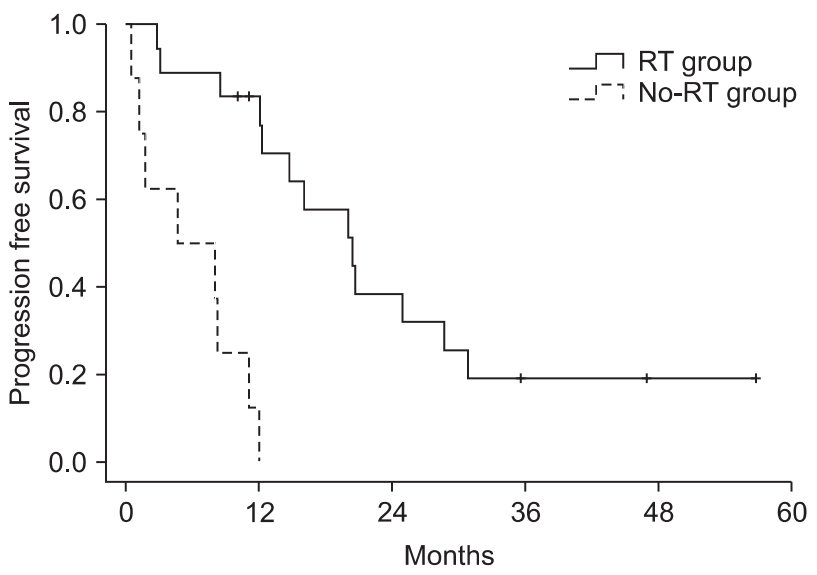

Fig. 1. Overall survival (A) and progression free survival (B) for the entire patients. RT, radiotherapy.

A

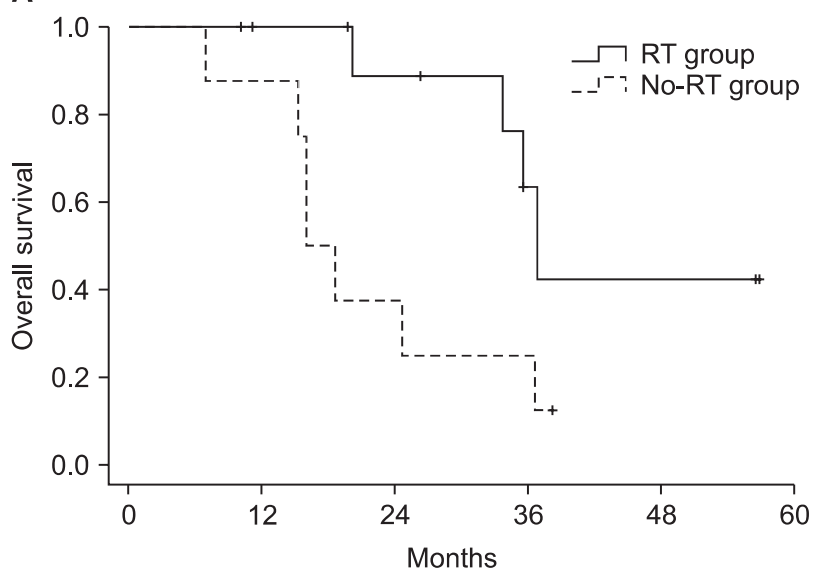

B

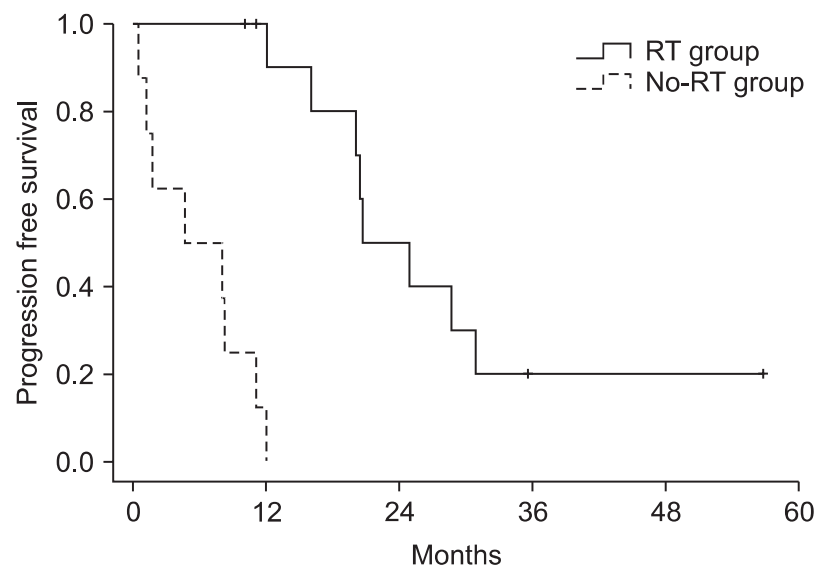

Fig. 2. Overall survival (A) and progression free survival (B) for the patients receiving upfront chemotherapy first without radiotherapy (RT).

PFS was 21 months in patients received RT and 5 months in patients without RT $(p<0.001)$ (Fig. 2). In addition, patients treated with upfront chemotherapy followed by RT showed significantly better OS than those treated with chemotherapy alone (median OS, 37 months vs. 16 months; $p=0.021$ ) (Fig. 2).

\section{Treatment toxicities}

During RT, acute gastrointestinal toxicity occurred in 14 patients (77.8\%), with RTOG grade 1 in 7 (38.9\%), and grade 2 in 7 (38.9\%). The most common side effects were nausea and abdominal discomfort, which were relieved in all patients with supportive medication. No grade 3 or more toxicity occurred during and after RT. In the course of salvage chemotherapy, 8 patients experienced grade $\geq 3$ neutropenia. Grade 3 stomatitis was observed in 2 patients. Other grade $\leq 2$ toxicities such as diarrhea, nausea, and anorexia were tolerable and alleviated after treatment.

\section{Discussion and Conclusion}

The aim of the present study is to evaluate the outcome of salvage treatment with or without RT in patients with RLNR after radical surgery in advanced gastric cancer. In this study, local failure rate was $27.8 \%$ in RT group, which was superior to that of $87.5 \%$ in no-RT group. Our data also demonstrate that OS and PFS were significantly associated with salvage RT, which might be attributed to low local failure rate.

Survival data have not been well-documented for patients 
with $L N$ recurrences after gastrectomy. However, the oncologic outcomes including OS and disease-free survival were generally disappointing. Ha and Kwon [10] found that most patients died within 1 year after diagnosis of recurrence and median survival times were 7, 9.5, and 12.5 months after the detection of peritoneal seeding, hematogenous metastasis, and locoregional recurrence, respectively. Eom et al. [11] reported that median survival time after recurrence was 7.4 months in the early (within 1 year after surgery) recurrence group and 14 months in the late recurrence group, which was statistically significant $(p<0.001)$. A retrospective study, which has similar study design of this work accruing patients with abdominal LN recurrences from gastric cancer, performed by Sun et al. [12] demonstrated that median survival was 11.4 months in the RT group and 4.8 months in the non-RT group ( $p=0.002$ ). They suggested that RT can reduce mortality of patients with $\mathrm{LN}$ recurrences after gastrectomy, and that LN metastasis from gastric cancer may be sensitive to RT. However, the overall survival of salvage RT group was comparable to other studies implementing systemic chemotherapy alone, implying that each of RT or chemotherapy may have effect on LN recurrences of gastric cancer. Median OS of the current study was 29 months which is better than those of historical controls better than those of historical controls [10-14]. Although many clinical or pathologic factors can contribute to the outcomes, aggressive treatment strategies including local and systemic modalities may be attributed to the outstanding outcomes. In the current study, salvage chemotherapy was routinely performed and RT was given in 18 of 26 patients (69\%).

No standard salvage treatment has been established yet for patients with LN recurrence after radical surgery for gastric cancer. In most cases, recurrences after gastrectomy are considered unsuitable for surgery and thus, chemotherapy has been the mainstay in the treatment. Koizumi et al. [13] demonstrated that combination chemotherapy with docetaxel, cisplatin, and S-1 is a well-tolerated and highly active regimen for advanced or recurrent gastric cancer, in which the median OS and PFS were 18.5 and 8.7 months, respectively. Choi et al. [14] performed a phase II study of docetaxel combined with oxaliplatin for recurrent gastric cancer. With the effectiveness of combination chemotherapy, the median OS and PFS were 13.8 and 5.3 months, respectively. These results are similar to the outcomes of patients with combination chemotherapy in our series, who did not receive RT.

With a development of systemic therapy, successful mana- gement of abdominal recurrence using local modalities has been reported. Kim et al. [15] found that patients with isolated para-aortic LN recurrence can be salvaged by stereotactic body RT without severe complications, in which the 3-year OS and PFS were 43\% and 29\%, respectively. Some studies showed that liver resection may offer the chance for cure even in patients with liver metastases of gastric cancer [16-18] These studies suggest that there might be a chance of cure or prolonging survival in highly selected cases with recurrent or metastatic disease by aggressive treatment including local modalities. In the same context, the results of the present study could provide additional rationale of active management of RLNR using salvage RT.

Despite of growing evidence that RT may help improving survival with enhancing local control in various circumstances of gastric cancer treatment, the role of perioperative RT is debatable. In a population-based study analyzing 47 thousand patients in the United States, RT demonstrated positive effect on survival with hazard radio of 0.8 [19]. Also, several recent studies showed promising results of perioperative RT, but this has not been widely accepted as a standard treatment in Asian countries, where gastric cancer is endemic and D2 LN dissection is routinely performed [20-22]. Paradoxically, in these Asian countries, RT may play a role in salvage setting. We expect that our data may contribute to improve upon therapeutic outcomes of locoregional recurrences of gastric cancer.

There were some limitations in this study. First, this is a retrospective study that many types of possible bias may affect treatment outcomes. For instance, heterogeneous chemotherapy regimens may influence patient's survival. Second, small number of patients also restricts statistical power.

In conclusion, our results show that salvage RT to RLNR from gastric cancer lowers local recurrence and may be associated with improved survival. As some patients still have cure chance after limited recurrences, active treatment is imperative to this population. Salvage RT combined with systemic chemotherapy may be an effective treatment managing RLNR from advanced gastric cancer. Further prospective studies with a large sample size are needed to draw definitive conclusions.

\section{Conflict of Interest}

No potential conflict of interest relevant to this article was reported. 


\section{References}

1. Korea Central Cancer Registry, National Cancer Center. Annual report of cancer statistics in Korea in 2009. Seoul: Ministry of Health and Welfare; 2011.

2. Jung KW, Park $\mathrm{S}$, Kong $\mathrm{HJ}$, et al. Cancer statistics in Korea: incidence, mortality, survival, and prevalence in 2009. Cancer Res Treat 2012;44:11-24.

3. Saito $H$, Fukumoto $Y$, Osaki $T$, et al. Prognostic significance of level and number of lymph node metastases in patients with gastric cancer. Ann Surg Oncol 2007;14:1688-93.

4. Chang JS, Lim JS, Noh SH, et al. Patterns of regional recurrence after curative D2 resection for stage III (N3) gastric cancer: implications for postoperative radiotherapy. Radiother Oncol 2012;104:367-73.

5. Schwarz RE, Zagala-Nevarez K. Recurrence patterns after radical gastrectomy for gastric cancer: prognostic factors and implications for postoperative adjuvant therapy. Ann Surg Oncol 2002;9:394-400.

6. Li JH, Zhang SW, Liu J, Shao MZ, Chen L. Review of clinical investigation on recurrence of gastric cancer following curative resection. Chin Med J (Engl) 2012;125:1479-95.

7. Song KY, Park SM, Kim SN, Park CH. The role of surgery in the treatment of recurrent gastric cancer. Am J Surg 2008;196:1922.

8. Macdonald JS, Smalley SR, Benedetti J, et al. Chemoradiotherapy after surgery compared with surgery alone for adenocarcinoma of the stomach or gastroesophageal junction. $\mathrm{N}$ Engl J Med 2001;345:725-30.

9. Japanese Gastric Cancer Association. Japanese Classification of Gastric Carcinoma (2nd English edition). Gastric Cancer 1998;1:10-24.

10. Ha TK, Kwon SJ. Clinicopathological characteristics for type of recurrence in gastric cancer patients. J Korean Gastric Cancer Assoc 2007;7:23-30.

11. Eom BW, Yoon $H$, Ryu KW, et al. Predictors of timing and patterns of recurrence after curative resection for gastric cancer. Dig Surg 2010;27:481-6.

12. Sun J, Sun YH, Zeng ZC, et al. Consideration of the role of radiotherapy for abdominal lymph node metastases in patients with recurrent gastric cancer. Int J Radiat Oncol Biol Phys
2010;77:384-91.

13. Koizumi $W$, Nakayama $N$, Tanabe $S$, et al. A multicenter phase II study of combined chemotherapy with docetaxel, cisplatin, and S-1 in patients with unresectable or recurrent gastric cancer (KDOG 0601). Cancer Chemother Pharmacol 2012;69: 407-13.

14. Choi YH, Oh SC, Kim JS, et al. A phase II study of docetaxel and oxaliplatin combination in recurrent gastric cancer patients after fluoropyrimidine and/or cisplatin adjuvant treatment: a Korean Cancer Study Group protocol ST06-02. Cancer Chemother Pharmacol 2012;70:665-72.

15. Kim MS, Yoo SY, Cho CK, et al. Stereotactic body radiotherapy for isolated para-aortic lymph node recurrence after curative resection in gastric cancer. J Korean Med Sci 2009;24:488-92.

16. Takemura N, Saiura A, Koga $R$, et al. Long-term outcomes after surgical resection for gastric cancer liver metastasis: an analysis of 64 macroscopically complete resections. Langenbecks Arch Surg 2012;397:951-7.

17. Makino $H_{1}$ Kunisaki $C$, Izumisawa $Y$, et al. Indication for hepatic resection in the treatment of liver metastasis from gastric cancer. Anticancer Res 2010;30:2367-76.

18. Tsujimoto $H_{\text {, Ichikura } T, O n o ~}$, et al. Outcomes for patients following hepatic resection of metastatic tumors from gastric cancer. Hepatol Int 2010;4:406-13.

19. Kunz PL, Gubens M, Fisher GA, Ford JM, Lichtensztajn DY, Clarke CA. Long-term survivors of gastric cancer: a California population-based study. J Clin Oncol 2012;30:3507-15.

20. Kim TH, Park SR, Ryu KW, et al. Phase 3 trial of postoperative chemotherapy alone versus chemoradiation therapy in stage III-IV gastric cancer treated with RO gastrectomy and D2 lymph node dissection. Int J Radiat Oncol Biol Phys 2012;84:e585-92.

21. Lee J, Lim DH, Kim S, et al. Phase III trial comparing capecitabine plus cisplatin versus capecitabine plus cisplatin with concurrent capecitabine radiotherapy in completely resected gastric cancer with D2 lymph node dissection: the ARTIST trial. J Clin Oncol 2012;30:268-73.

22. Lee DJ, Sohn TS, Lim DH, et al. Phase I study of neoadjuvant chemoradiotherapy with S-1 and oxaliplatin in patients with locally advanced gastric cancer. Cancer Chemother Pharmacol 2012;69:1333-8. 